\title{
Cognitive Strategies in Student Team Projects
}

\author{
Nick Jensen, Brent Fukuda, Philip Daum, Students, $4^{\text {th }}$ year \\ Carmen Ciubotariu and Garwin Hancock \\ Department of electrical and Computer Engineering, University of Calgary, \\ T2N 1N4 Calgary, Alberta, Canada \\ cciubota@ucalgary.ca
}

This paper illustrates the learning benefits and the collective efficacy of student project teams in engineering design education.

We describe the impact of learning for a fourth year design project on student lifetime knowledge at both ends: project builders (fourth year students enrolled in the three programs of Electrical, Computer and Software engineering of the Department of Electrical and Computer Engineering of the University of Calgary) and product users- all students taking electric circuits laboratories (for our programs or from the Faculty of Engineering-common core).

Much understanding about learning and cognition has been achieved during the cognitive analysis of the course outcomes for the first course on digital circuits offered in the Department of Electrical and Computer Engineering of the University of Calgary. As a result, we present cognitive strategies and their use in the introduction of simple digital circuits (flip-flops) and the design of a traffic light controller.

We have developed a virtual laboratory with digital circuits and instrumentation components is a first attempt to motivate students to do the pre-lab exercises for digital and electric circuits and reuse them to refresh their training in using the appropriate functions of the buttons and devices needed for any electronic data generation and acquisition. The components of this educational environment have been created based on the criteria of professionalism stated by the guidelines of the Association of Professional Engineers, Geophysicists and Geologists of Alberta (APEGGA) and the University of Calgary learning plan.

Over the past four years, six student teams have undertaken and successfully accomplished design projects which produced the main components of the departmental virtual laboratory: real-time control of electronic instruments and of a traffic light, traffic signal simulation with a digitized voice synthesis tool and operational simulation of simple finite state machines. The educational site integrates exercises with progressive degree of difficulty, from simple gates to digital controllers. The students are invited to take a virtual visit of the laboratory rooms in which they will perform course required experiments in order to easier locate the devices to be used as well as operate them appropriately.

For project completion, the teams have been trained to adopt a framework for implementing a hierarchical model of learning as the basis for developing skills and methods.

Keywords: learning, concept mapping, knowledge mapping, inquiry-based learning, simulation, remote control instrumentation

Abstract submitted for the Second CDEN international Conference, Kananaskis, July 18-20, 2005

Author for correspondence: Carmen, cciubota@ucalgary.ca 